\title{
The Efficacy and Safety of Elobixibat for the Elderly with Chronic Constipation: A Multicenter Retrospective Cohort Study
}

\author{
Akira Tomie, ${ }^{1}$ Naohisa Yoshida $\mathbb{D}^{2},{ }^{2}$ Munehiro Kugai, ${ }^{3}$ Ryohei Hirose $\mathbb{D}^{2},{ }^{2}$ Osamu Dohi $\mathbb{D},{ }^{2}$ \\ Ken Inoue, ${ }^{2}$ Kotaro Okuda, ${ }^{4}$ Takayuki Motoyoshi, ${ }^{5}$ Kohei Fukumoto, ${ }^{6}$ Yoshikazu Inagaki, ${ }^{7}$ \\ Hiroyuki Yoriki, ${ }^{8}$ Yutaka Inada, ${ }^{9}$ Takashi Okuda, ${ }^{9}$ Daisuke Hasegawa, ${ }^{10}$ Kiyoshi Ogiso $\mathbb{D}^{11}$ \\ Takaaki Murakami $\mathbb{D}^{12},{ }^{12}$ Koichi Soga $\mathbb{D}^{13},{ }^{13}$ Rafiz Abdul Rani, ${ }^{14}$ Norimasa Yoshida, ${ }^{1}$ \\ and Yoshito Itoh $^{2}$
}

${ }^{1}$ Department of Gastroenterology, Saiseikai Kyoto Hospital, Kyoto, Japan

${ }^{2}$ Department of Molecular Gastroenterology and Hepatology, Kyoto Prefectural University of Medicine, Graduate School of Medical Science, Kyoto, Japan

${ }^{3}$ Department of Gastroenterology, Akashi City Hospital, Hyogo, Japan

${ }^{4}$ Department of Gastroenterology, Kyoto Kujo Hospital, Kyoto, Japan

${ }^{5}$ Department of Gastroenterology, Kyoto City Hospital, Kyoto, Japan

${ }^{6}$ Department of Gastroenterology, Nara City Hospital, Nara, Japan

${ }^{7}$ Department of Gastroenterology, Nishijin Hospital, Kyoto, Japan

${ }^{8}$ Department of Gastroenterology, Otsu City Hospital, Shiga, Japan

${ }^{9}$ Department of Gastroenterology, Fukuchiyama City Hospital, Kyoto, Japan

${ }^{10}$ Department of Gastroenterology, Ayabe City Hospital, Kyoto, Japan

${ }^{11}$ Department of Gastroenterology, Osaka General Hospital of West Japan Railway Company, Osaka, Japan

${ }^{12}$ Department of Gastroenterology, Japan Community Health Care Organization, Kyoto Kuramaguchi Medical Center, Kyoto, Japan

${ }^{13}$ Department of Gastroenterology, Omihachiman Community Medical Center, Shiga, Japan

${ }^{14}$ Gastroenterology Unit, Faculty of Medicine, Universiti Teknologi MARA, Selangor, Malaysia

Correspondence should be addressed to Naohisa Yoshida; naohisa@koto.kpu-m.ac.jp

Received 10 February 2020; Revised 1 April 2020; Accepted 6 April 2020; Published 28 April 2020

Academic Editor: Raquel Martín-Venegas

Copyright (C) 2020 Akira Tomie et al. This is an open access article distributed under the Creative Commons Attribution License, which permits unrestricted use, distribution, and reproduction in any medium, provided the original work is properly cited.

Backgrounds and Aims. Elobixibat is a bile acid transporter inhibitor indicated for constipation. Previous studies were performed mainly for the nonelderly and were biased to female. We analyzed the efficacy of elobixibat also for the elderly and male. Materials and Methods. This was a multicenter retrospective cohort study. The subjects were patients aged $\geq 20$ years treated for chronic constipation from May 2018 to November 2019 at 12 related institutions. Patients were divided into $\leq 74$ years and $\geq 75$ years old. Elobixibat at $10 \mathrm{mg} /$ day was prescribed for two weeks. We then analyzed the discontinuation due to ineffectiveness, change of spontaneous bowel movements (SBM), stool consistency, the time until the first SBM, adverse events, and effect-related factors. Results. There were 140 cases (61 males) evaluated, with an average age of $72.1 \pm 13.6$ years ( $\leq 74$ years: 71 cases; $\geq 75$ years: 69 cases). The discontinuation rate was 7.9\%. The SBM (times/week) increased from 2.86 to $6.08(p<0.001)$. The overall SBM improvement rate was $74.0 \%$ ( $\leq 74$ years: $78.2 \%$ vs. $\geq 75$ years: $68.9 \%, p=0.31$; male: $75.0 \%$ vs. female: $73.3 \%, p=0.78$ ). The overall improvement rate of stool consistency was $59.6 \%$ ( $\leq 74$ years: $62.9 \%, \geq 75$ years: $56.1 \%, p=0.42$ ). The time until the first SBM (hours) for those $\leq 74$ years and $\geq 75$ years was $17.2 \pm 14.3$ and $11.2 \pm 8.4(p=0.04)$. Adverse event rates for those $\leq 74$ years and $\geq 75$ years were $28.2 \%$ and $10.1 \%(p<0.01)$. There were no significant effect-related factors for gender, age, and use of laxatives. Conclusions. Short-period elobixibat is shown to be effective also for the elderly and male. 


\section{Introduction}

According to a national survey conducted in 2013, the prevalence of chronic constipation in Japan was $2.6 \%$ for male and $4.9 \%$ for female. It increases with age for both genders, with the prevalence rate assumed at approximately $10 \%$ for those who are $\geq 75$ years old [1]. The prevalence in Western countries has been reported to be approximately $15 \%$ throughout the entire age group with the frequency also increasing according to age [2-4]. It has been reported that QOL was lower in patients with chronic constipation compared to healthy individuals, indicating the importance of treatment [5].

In Japan, magnesium oxide and anthraquinolone stimulant laxatives have been widely used for chronic constipation. The guideline for the medical treatment of constipation set by the American College of Gastroenterology proposes lifestyle habit guidance and the administration of osmotic laxatives, similar to the Japanese guidelines [6-8]. In these 10 years, several new drugs for chronic constipation were launched. Lubiprostone, a selective chloride channel activator, and linaclotide, a guanylate cyclase $\mathrm{C}$ receptor agonist, were introduced to the market in 2012 and 2017, respectively [9-16]. Elobixibat, a bile acid transporter inhibitor, was launched in 2018 in Japan as a world's first. It increases bile acid influx into the colon by inhibiting bile acid transporters expressed on epithelial cells of the terminal ileum, therefore suppressing bile acid reabsorption, increasing water secretion into the colorectum, and increasing gastrointestinal motility. Some researches had been performed on elobixibat in Sweden and United States around 2011 and followed by several clinical trials in Japan around 2018 [17-23]. However, studies to date have included safety studies with less than 50 cases, and even when more than 100 cases were scrutinized, the average age of subjects was approximately 50 years and $75 \%$ or more were female.

In this study, we investigated the efficacy and safety of elobixibat for chronic constipation in $\geq 100$ cases including the elderly and male.

\section{Materials and Methods}

We conducted a multicenter, single-arm, and retrospective cohort study. The subjects were 157 patients with chronic constipation who were prescribed elobixibat, from May 2018 to November 2019, at a total of 12 related institutions (Saiseikai Kyoto Hospital, Kyoto Prefectural University of Medicine, Akashi City Hospital, Kyoto Kujo Hospital, Kyoto City Hospital, Nara City Hospital, Nishijin Hospital, Otsu City Hospital, Fukuchiyama City Hospital, Ayabe City Hospital, Osaka General Hospital of West Japan Railway Company, and JCHO Kyoto Kuramaguchi Medical Center). The eligibility criteria were patients over the age of 20 and suffering from two or more of the following six criteria of chronic constipation under the Rome IV standard: (1) straining, (2) hard stool, (3) residual stool feeling, (4) occlusion feeling, (5) manual bowel movement performed at a frequency of $25 \%$ or more of bowel movements, and (6) frequency of bowel movements of $<3$ times a week [24]. The term "chronic" was defined as "symptoms that have been present for at least 6 months and the criteria for 'constipation' described above have been met for at least 3 months." We excluded patients with severe cardiopulmonary, hepatic, or renal disease. We also excluded cases in which patients could not continue taking elobixibat due to factors other than ineffectiveness or side effects. Excluded were also cases at which the data of all three items such as stool frequency, stool consistency, and side effects, were unavailable. Regarding the way of administration of elobixibat, oral administration was initiated at $10 \mathrm{mg}$ once a day before breakfast, but it was possible to reduce the dose to $5 \mathrm{mg}$ once a day before breakfast or increase the dose to $15 \mathrm{mg}$ once a day before breakfast depending on the symptoms. Elobixibat was continued for 2 weeks. This study was conducted based on the World Medical Association Helsinki Declaration and approved by the ethics committee of the representative facility, Saiseikai Kyoto Hospital. This study was retrospective in setting, and an opt-out about the study to the patients was performed in the representative facility.

The evaluation items for this study were the discontinuance rate of elobixibat due to ineffectiveness, adverse events, and the number of spontaneous bowel movements (SBM) one week before and after the administration of elobixibat. We analyzed SBM for the nonelderly aged $\leq 74$ years and elderly patients aged $\geq 75$ years as well as for male and female. Additionally, the improvement rate for SBM was calculated. The number of SBM referred to bowel movements that occurred without a laxative/enema or manual evacuation. The improvement of SBM was defined as the increase of $\geq 1$ bowel movement a week from the baseline and also having $\geq 3$ times bowel movement a week based on a previous report [20]. The changes in stool consistency according to the Bristol stool chart were also analyzed in the nonelderly aged $\leq 74$ years, elderly patients aged $\geq 75$ years, male, and female [25]. The Bristol stool chart is a global standard for evaluating stool shape. The subjects evaluated themselves on a 7-point scale. Types 1 and 2 of this scale are hard stools, whereas types 6 and 7 are loose stools. With respect to the stool consistency, "a change to types 3-5" was considered as having improved. The times until the first SBM after the administration of elobixibat and the presence of SBM within 24 hours were analyzed. Adverse events were also examined. Regarding the analysis of effect-related factors, subjects were divided into two groups, with or without improvement in SBM, in order to conduct a comparative study on gender, age ( $\leq 74$ years vs. $\geq 75$ years), concomitant use of laxatives, concomitant use of stimulant laxatives, elobixibat dose $(5 \mathrm{mg}$ /day vs. $10 \mathrm{mg}$ /day vs. $15 \mathrm{mg} /$ day), the presence of underlying diseases (dyslipidemia, diabetes, hepatic disorder, hypothyroidism, and Parkinson's disease), and other concomitant drugs (antacids, antidepressants, calcium antagonists, Parkinson's disease drugs, narcotics for cancer pain, and ursodeoxycholic acid). We obtained all of patients' information including the evaluation items about elobixibat from the medical records in each hospital. Some facilities utilized a questionnaire after the administration of elobixibat while others did not. It was at the discretion of each facility and doctor. 


\section{Statistical Analysis}

The $U$ test was used to compare continuous variables for changes in the number of SBM before and after the administration of elobixibat. The improvement rate of SBM, improvement rate of stool consistency, age comparison of the SBM rate within 24 hours, and comparison of background factors depending on whether or not the number of SBM had improved were determined by the chi-squared test and Fisher's exact test (SPSS version 22.0 for Windows; IBM Japan, Ltd., Tokyo, Japan). $p<0.05$ was considered significant for all statistical analyses.

\section{Results}

We analyzed 140 cases out of the 157 patients. SBM frequency, stool consistency, and side effects were evaluated (Table 1). The gender was 61 males (43.6\%) and 79 females (56.4\%) with the average age of $72.1( \pm 13.6)$ years. In regard to age, 71 cases $(50.7 \%)$ were $\leq 74$ years old and 69 cases $(49.3 \%)$ were $\geq 75$ years old. Seventy-six patients $(54.3 \%)$ used other laxatives in combination with elobixibat. Ninety-two patients $(65.7 \%)$ continued taking $10 \mathrm{mg}$ elobixibat per day until the second week of administration with 27 patients (19.3\%) decreasing the dose to $5 \mathrm{mg}, 10$ patients $(7.1 \%)$ increasing the dose to $15 \mathrm{mg}$, and 11 patients (7.9\%) discontinuing. In these 11 cases, 6 were due to adverse events while 5 were due to ineffectiveness.

For the 104 evaluated cases of SBM, the frequency of SBM (average $\pm \mathrm{SD}$ ) in the week prior to elobixibat was $2.86 \pm 1.77$ times/week and the frequency increased to $6.08 \pm 4.65$ times/ week after it $(p<0.001)$ (Figure 1$)$. The frequency (times/ week) increased from $2.82 \pm 1.85$ to $6.31 \pm 4.77$ in the group of $\leq 74$ years old $(p<0.001)$, and it also increased from 2.90 \pm 1.68 to $5.82 \pm 4.54$ times/week in the group of $\geq 75$ years old $(p<0.001)$, indicating improvements regardless of age.

With respect to the improvement rate in the frequency of SBM, 77 cases were found effective (74.0\%) among all 104 evaluated cases (Figure 2). The improvement rate was $78.2 \%$ for $\leq 74$ years and $69.4 \%$ for $\geq 75$ years with no difference in terms of age $(p=0.31)$. With respect to the effect of gender, the improvement rate was $75.0 \%$ for male and $73.3 \%$ for female $(p=0.78)$.

Regarding the stool consistency improvement rate, 81 (59.6\%) of the 136 cases that could be evaluated showed improvements of stool consistency (Figure 2). It was $62.9 \%$ for those $\leq 74$ years and $56.1 \%$ for those $\geq 75$ years, indicating no difference by age $(p=0.42)$. With respect to the effect on male and female, it was $54.2 \%$ for male and $63.6 \%$ for female $(p=0.18)$.

The mean time to first SBM (hours) after taking elobixibat was $14.8 \pm 12.6$ (Table 2). Regarding age, the time to first SBM was significantly shorter for those $\geq 75$ years compared to those $\leq 74$ years $(11.2 \pm 8.4$ vs. $17.2 \pm 14.3, p=0.04)$. The SBM rate within 24 hours after taking elobixibat was 85 $(78.7 \%)$ out of the 108 evaluable patients (Table 2). It was $78.3 \%$ for $\leq 74$ years and $79.3 \%$ for $\geq 75$ years, indicating no difference in terms of age $(p=0.92)$.

Adverse events were observed in 27 (19.3\%) of all 140 cases, described as follows: 16 cases (11.4\%) of diarrhea, 12
TABle 1: Patient characteristics.

\begin{tabular}{lc}
\hline Number of cases & 140 \\
\hline Gender, $n(\%)$, male $:$ female & $61: 79(43.6: 56.4)$ \\
Age, average \pm SD & $72.1 \pm 13.6$ \\
Age distribution, $n(\%), \leq 74: \geq 75$ & $71: 69(50.7: 49.3)$ \\
Laxative combination, $n(\%)$ & $76(54.3)$ \\
Irritant laxative combination, $n(\%)$ & $34(24.3)$ \\
Elobixibat dose (mg), $n(\%)$, & $27: 92: 10: 11$ \\
$5: 10: 15:$ discontinuation & $(19.3: 65.7: 7.1: 7.9)$ \\
Underlying disease, $n(\%)$ & \\
Dyslipidemia & $42(30.0)$ \\
Diabetes & $25(17.9)$ \\
Hepatic disorder & $10(7.1)$ \\
Hypothyroidism & $6(4.3)$ \\
Parkinson's disease & $5(3.6)$ \\
Concomitant medication, $n(\%)$ & \\
Antacids & $55(39.3)$ \\
Antidepressants & $14(10.0)$ \\
Calcium antagonists & $11(7.9)$ \\
Parkinson's disease drugs & $5(3.6)$ \\
Opioids & $4(2.9)$ \\
Ursodeoxycholic acid & $3(2.1)$ \\
\hline
\end{tabular}

SD: standard deviation.

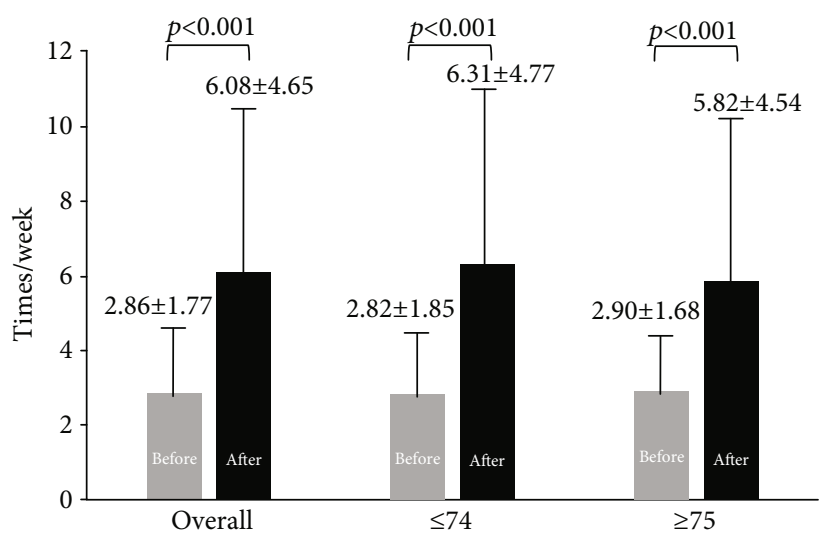

FIGURE 1: Changes of weekly spontaneous bowel movements after elobixibat with regard to age.

cases $(8.6 \%)$ of abdominal pain, 4 cases $(2.9 \%)$ of abdominal distension, and 1 case $(0.7 \%)$ of nausea. Some cases had more than 2 complaints. The rates for $\geq 75$ years and $\leq 74$ years were $10.1 \%$ and $28.2 \%(p<0.01)$, respectively. The frequency of adverse events by gender was $16.4 \%$ for male and $21.5 \%$ for female $(p=0.36)$.

Regarding the analysis of effect-related factors, a comparison was made of background factors, depending on whether SBM had improved or not (Table 3). There was no significant difference with respect to gender, age ( $\leq 74$ years vs. $\geq 75$ years), concomitant use of laxatives, concomitant use of stimulant laxatives, elobixibat dose, presence of underlying diseases (diabetes, hypothyroidism, Parkinson's disease, dyslipidemia, and hepatic disorder), and the use of concomitant 

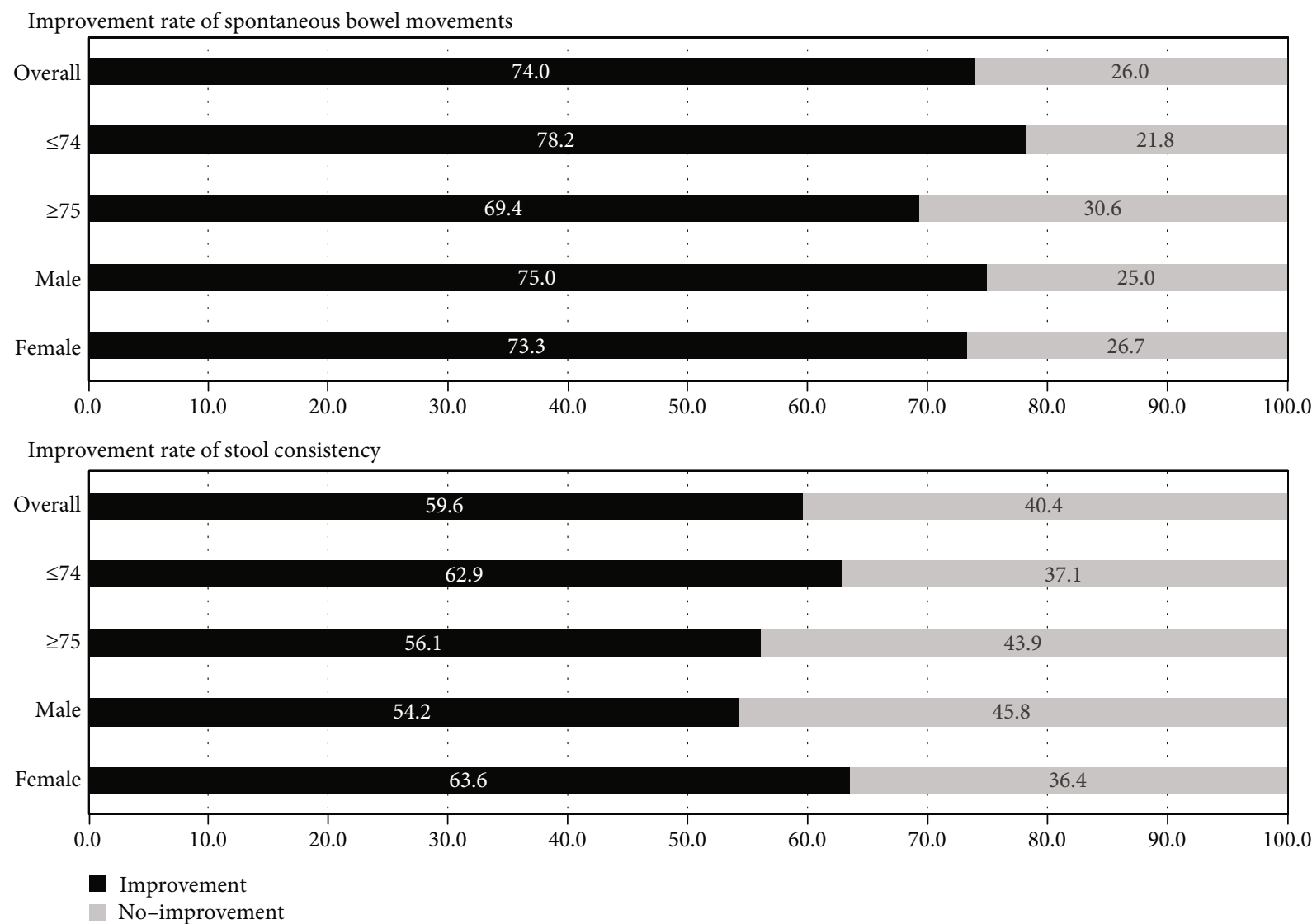

FIGURE 2: Improvement rate of spontaneous bowel movements and stool consistency after prescription of elobixibat with regard to age and sex.

TABLE 2: Time to first spontaneous bowel movements and rate of spontaneous bowel movement within 24 hours after prescription of elobixibat.

Time to first spontaneous bowel movements (hr) (mean \pm SD)

$$
\begin{gathered}
\text { Overall }(N=95) \\
\quad \leq 74(N=56) \\
\geq 75(N=39)
\end{gathered}
$$

$14.8 \pm 12.6$

$17.2 \pm 14.3^{*}$

$11.2 \pm 8.4^{* *}$

Rate of spontaneous bowel movement within 24 hours

\begin{tabular}{cc} 
Overall $(N=95)$ & $78.7 \%$ \\
$\leq 74(N=56)$ & $78.3 \%{ }^{* * *}$ \\
$\geq 75(N=39)$ & $79.3 \%{ }^{* * * *}$ \\
\hline SD: standard deviation. ${ }^{*}$ vs. ${ }^{* *} p=0.04,{ }^{* * *}$ vs. ${ }^{* * * *} p=0.92$.
\end{tabular}

drugs (antacids, Parkinson's disease drugs, antidepressants, calcium antagonists, narcotics for cancer pain, and ursodeoxycholic acid).

\section{Discussion}

The efficacy and safety of elobixibat in $\geq 100$ cases including the elderly and male were verified in this study. The average age of patients was $72.1 \pm 13.6$ years, and it is the oldest among all elobixibat studies to date. Additionally, regarding gender, our study included $43.6 \%$ male subjects (61 cases), being the highest number to date. Regarding the improvement rate of SBM with elobixibat, the rate was $78.2 \%$ and there was no significant difference between those $\leq 74$ years old and those $\geq 75$ years old. The rates of adverse events for $\leq 74$ years old and $\geq 75$ years old were $28.2 \%$ and $10.1 \%$ $(p<0.01)$, respectively. Additionally, there were no significant differences on the improvement rates of SBM, stool consistency, and adverse events between male and female.

In regard to case characteristics, in two studies to verify safety of elobixibat in Sweden and the United States, the number of cases was only 30 and 36 , respectively $[17,18]$. The only report involving over 100 cases in the United States included 190 subjects; however, the subjects were mainly nonelderly (mean age: 48.1 years) and 90\% were female [19]. One report in Japan verified the safety of elobixibat with multiple doses in 59 cases (mean age: $35.4 \pm 10.8$ years) [21]. Two reports in Japan indicated short-term results from twoweek administration: one included 163 patients with an average age of 43.4-46.1 years, among which $88 \%$ were female; and the other included 132 patients with an average age of 43.0 years, among which $83 \%$ were female [20, 22]. Furthermore, in a Japanese study looking at the long-term results of 52 -week treatment, the subjects were 340 patients averaging 43.9 years, among which $83 \%$ were female (283 patients), which was biased [20]. Our case's number in thsi study is 140 and included $56.4 \%$ females and 69 patients $\geq 75$ years old. This is the largest study, which was not biased to either nonelderly or female. 
TABLE 3: The comparison between cases with improvement of spontaneous bowel movements and cases without it.

\begin{tabular}{lccc}
\hline & $\begin{array}{c}\text { With improved } \\
\text { bowel movements } \\
(n=77)\end{array}$ & $\begin{array}{c}\text { Without improved } \\
\text { bowel movement } \\
(n=27)\end{array}$ & $p$ value \\
\hline Gender, $n$ (\%) & $33(42.9)$ & $11(40.7)$ & 0.85 \\
Male & $44(57.1)$ & $16(59.3)$ & \\
Female & & & 0.31 \\
Age, $n$ (\%) & $43(55.8)$ & $12(44.4)$ & 0.52 \\
$\leq 74$ & $34(48.1)$ & $15(55.6)$ & 0.61 \\
$\geq 75$ & $43(44.8)$ & $17(63.0)$ & 0.53 \\
Laxative combination, $n$ (\%) & $21(27.3)$ & $6(22.2)$ & \\
Irritant laxative combination, $n$ (\%) & & & \\
Elobixibat dose, $n$ (\%) & $18(23.4)$ & $4(14.8)$ & 0.97 \\
5 mg & $51(66.2)$ & $21(77.8)$ & 0.80 \\
10 mg & $8(10.4)$ & $2(7.4)$ & 0.46 \\
15 mg & & & 0.23 \\
Underlying disease, $n$ (\%) & $26(33.8)$ & $9(33.3)$ & 0.30 \\
Dyslipidemia & $16(20.8)$ & $5(18.5)$ & \\
Diabetes & $3(3.9)$ & $2(7.4)$ & 0.97 \\
Hepatic disorder & $4(5.2)$ & $0(0)$ & 0.41 \\
Hypothyroidism & $3(3.9)$ & $0(0)$ & 0.65 \\
Parkinson's disease & & & 0.30 \\
Concomitant medication, $n$ (\%) & $31(40.3)$ & $11(40.7)$ & 0.40 \\
Antacids & $7(9.1)$ & $4(14.8)$ & $2(7.4)$ \\
Antidepressants & $8(10.4)$ & $0(0)$ & $0(0)$ \\
Calcium antagonists & $3(3.9)$ & & \\
Parkinson's disease drugs & $2(2.6)$ & & \\
Ursodeoxycholic acid & & & \\
\hline
\end{tabular}

With respect to the efficacy of elobixibat, the improvement rate of SBM was $94.0 \%$ at a $10 \mathrm{mg}$ oral dose in a previous Japanese study, which was higher than $74.0 \%$ noted in our study which includes the elderly (median age: $72.1 \pm$ 13.6 years, female rate: 56.4\%) [20]. We showed that the improvement rates of SBM with regard to age were $78.2 \%$ for patients $\leq 74$ years old and $69.4 \%$ for $\geq 75$ years old. Additionally, we showed that the rates with regard to gender were $75.0 \%$ for male and $73.3 \%$ for female. The number of SBM after elobixibat administration was 6.40 times/week in the Nakajima et al. study, which was also slightly higher than 6.08 times/week in our study [20]. There was no difference between patients $\leq 74$ years old and $\geq 75$ years old. Thus, this is the first report, which showed the efficacy of elobixibat related to age and gender. Our study could indicate that elobixibat is effective for the elderly and male similar to the nonelderly and female. However, in our study, the cases' number was still inadequate, and moreover, it was in a retrospective setting. A large-scale prospective study that is not biased to either age or gender should be performed in the future.

Regarding the first SBM, there was a unique result in our study. Although it was reported that the time (median) to the first SBM was as short as 5.2 hours, the mean time was as long as 14.8 hours in our study [20]. This is probably due to the difference of the characteristics of the patients. The patients in our study were older, and $54.3 \%$ of the patients received other laxatives. Thus, patients with a slightly severe constipation compared with those of previous studies were enrolled. Our study also showed that the time in patients aged $\geq 75$ years old was significantly shorter than those aged $\leq 74$ years old, although the SBM/week after elobixibat was slightly smaller in patients aged $\geq 75(5.82 \pm 4.54)$ than patients aged $\leq 74(6.31 \pm 4.77)$. We suspected that the possible reason for this was that the first SBM was different from the SBM/week affected by many factors including age, sex, laxative, and severity of baseline constipation. Another plausible reason is that the result was due to the retrospective setting of this study. The time was analyzed based on patients' memory, and some might reply with uncertainty. These data should be analyzed prospectively in the future.

With respect to the side effects, two previous studies in Japan reported that the rates of abdominal pain were $26 \%$ and $18.8 \%$ while those of diarrhea were $5 \%$ and $13.0 \%$ when $10 \mathrm{mg}$ of elobixibat was administered, whereas the frequency in our study involving the elderly indicated $8.6 \%$ for abdominal pain and $11.4 \%$ for diarrhea, showing no significant difference, and as such, it should be considered highly safe among both the elderly and the nonelderly $[20,22]$.

We also examined effect-related factors that were not verified in previous reports. There was no difference to the treatment effect that can be attributed to combination with other laxatives including irritant laxatives, the underlying disease, 
or the use of concomitant medications. This indicates that the effect was obtained regardless of background factors.

There were several limitations associated with the present study. This was a single-arm retrospective study. There is a possibility that the diagnosis of chronic constipation and the indication of this drug prescription may vary among prescribing physicians.

\section{Conclusion}

In more than 100 clinical cases including the elderly and male, elobixibat for chronic constipation was confirmed to be useful and safe over a short period of time, regardless of age, gender, underlying disease, or concomitant medication.

\section{Data Availability}

The patient data used to support the findings of this study are available from the corresponding author upon request. However, some data may be restricted by the institutional review board of Saiseikai Kyoto Prefectural Hospital.

\section{Conflicts of Interest}

The authors declare that they have no conflicts of interest.

\section{Acknowledgments}

We would like to extend our special appreciation to Dr. Yasutaka Morimoto, Dr. Tomoyuki Ohno, Dr. Ritsu Yasuda, Dr. Naoki Wakabayashi, members of the Kyoto Improvement of Colonoscopy Seminar (KICS) group, and all medical members at Molecular Gastroenterology and Hepatology in the Kyoto Prefectural University of Medicine.

\section{References}

[1] S. Bureau, "Ministry of Internal Affairs and Communications," http://www.stat.go.jp/english/index.html.

[2] P. D. R. Higgins and J. F. Johanson, "Epidemiology of constipation in North America: a systematic review," The American Journal of Gastroenterology, vol. 99, no. 4, pp. 750-759, 2004.

[3] E. Bouras and M. Vazquez-Roque, "Epidemiology and management of chronic constipation in elderly patients," Clinical Interventions in Aging, vol. 10, pp. 919-930, 2015.

[4] J. F. Johanson, A. Sonnenberg, and T. R. Koch, "Clinical epidemiology of chronic constipation," Journal of Clinical Gastroenterology, vol. 11, no. 5, pp. 525-536, 1989.

[5] A. Glia and G. Lindberg, "Quality of life in patients with different types of functional constipation," Scandinavian Journal of Gastroenterology, vol. 32, no. 11, pp. 1083-1089, 1997.

[6] American College of Gastroenterology Chronic Constipation Task Force, "An evidence-based approach to the management of chronic constipation in North America," The American Journal of Gastroenterology, vol. 100, pp. S1-S22, 2005.

[7] C. Nyberg, J. Hendel, and O. H. Nielsen, "The safety of osmotically acting cathartics in colonic cleansing," Nature Reviews. Gastroenterology \& Hepatology, vol. 7, no. 10, pp. 557-564, 2010.
[8] A. E. Bharucha, J. H. Pemberton, and G. R. Locke III, "American Gastroenterological Association technical review on constipation," Gastroenterology, vol. 144, no. 1, pp. 218-238, 2013.

[9] J. F. Johanson, D. Morton, J. Geenen, and R. Ueno, "Multicenter, 4-week, double-blind, randomized, placebo-controlled trial of lubiprostone, a locally-acting type- 2 chloride channel activator, in patients with chronic constipation," The American Journal of Gastroenterology, vol. 103, no. 1, pp. 170-177, 2008.

[10] J. F. Johanson and R. Ueno, "Lubiprostone, a locally acting chloride channel activator, in adult patients with chronic constipation: a double-blind, placebo-controlled, dose-ranging study to evaluate efficacy and safety," Alimentary Pharmacology \& Therapeutics, vol. 25, no. 11, pp. 1351-1361, 2007.

[11] S. Fukudo, M. Hongo, H. Kaneko, M. Takano, and R. Ueno, "Lubiprostone Increases Spontaneous Bowel Movement Frequency and Quality of Life in Patients With Chronic Idiopathic Constipation," Clinical Gastroenterology and Hepatology, vol. 13, no. 2, pp. 294-301.e5, 2015.

[12] S. Fukudo, M. Hongo, H. Kaneko, and R. Ueno, "Efficacy and safety of oral lubiprostone in constipated patients with or without irritable bowel syndrome: a randomized, placebocontrolled and dose-finding study," Neurogastroenterology and Motility, vol. 23, no. 6, p. 544, 2011.

[13] W. D. Chey, A. J. Lembo, B. J. Lavins et al., "Linaclotide for irritable bowel syndrome with constipation: a 26-week, randomized, double-blind, placebo-controlled trial to evaluate efficacy and safety," The American Journal of Gastroenterology, vol. 107, no. 11, pp. 1702-1712, 2012.

[14] S. Rao, A. J. Lembo, S. J. Shiff et al., "A 12-week, randomized, controlled trial with a 4 -week randomized withdrawal period to evaluate the efficacy and safety of linaclotide in irritable bowel syndrome with constipation," The American Journal of Gastroenterology, vol. 107, no. 11, pp. 1714-1725, 2012.

[15] A. J. Lembo, H. A. Schneier, S. J. Shiff et al., “Two Randomized trials of Linaclotide for chronic constipation," The New England Journal of Medicine, vol. 365, no. 6, pp. 527-536, 2011.

[16] A. J. Lembo, C. B. Kurtz, J. E. MacDougall et al., "Efficacy of Linaclotide for Patients With Chronic Constipation," Gastroenterology, vol. 138, no. 3, pp. 886-895.e1, 2010.

[17] M. Simrén, A. Bajor, P.-G. Gillberg, M. Rudling, and H. Abrahamsson, "Randomised clinical trial: the ileal bile acid transporter inhibitor a3309 vs. placebo in patients with chronic idiopathic constipation a double-blind study," Alimentary Pharmacology \& Therapeutics, vol. 34, no. 1, pp. 4150, 2011.

[18] B. S. Wong, M. Camilleri, S. McKinzie, D. Burton, H. Graffner, and A. R. Zinsmeister, "Effects of a 3309, an ileal bile acid transporter inhibitor, on colonic transit and symptoms in females with functional constipation," The American Journal of Gastroenterology, vol. 106, no. 12, pp. 2154-2164, 2011.

[19] W. D. Chey, M. Camilleri, L. Chang, L. Rikner, and H. Graffner, "A randomized placebo-controlled phase IIb trial of a 3309, a bile acid transporter inhibitor, for chronic idiopathic constipation," The American Journal of Gastroenterology, vol. 106, no. 10, pp. 1803-1812, 2011.

[20] A. Nakajima, M. Seki, S. Taniguchi et al., "Safety and efficacy of elobixibat for chronic constipation: results from a randomised, double-blind, placebo-controlled, phase 3 trial and an openlabel, single-arm, phase 3 trial," The Lancet Gastroenterology \& Hepatology, vol. 3, no. 8, pp. 537-547, 2018. 
[21] Y. Kumagai, H. Amano, Y. Sasaki et al., "Effect of single and multiple doses of elobixibat, an ileal bile acid transporter inhibitor, on chronic constipation: a randomized controlled trial," British Journal of Clinical Pharmacology, vol. 84, no. 10, pp. 2393-2404, 2018.

[22] A. Nakajima, M. Seki, and S. Taniguchi, "Determining an optimal clinical dose of elobixibat, a novel inhibitor of the ileal bile acid transporter, in Japanese patients with chronic constipation: a phase II, multicenter, double blind, placebo controlled randomized clinical trial," Journal of Gastroenterology, vol. 53, no. 4, pp. 525-534, 2018.

[23] A. Nakajima, S. Taniguchi, S. Kurosu, P. G. Gillberg, J. P. Mattsson, and M. Camilleri, "Efficacy, long-term safety, and impact on quality of life of elobixibat in more severe constipation: post hoc analyses of two phase 3 trials in Japan," Neurogastroenterology and Motility, vol. 31, no. 5, 2019.

[24] D. A. Drossman, L. Chang, W. D. Chey et al., Rome IV Functional Gastrointestinal Disorders, Rome Foundation, INC., 4th edition, 2016.

[25] S. J. Lewis and K. W. Heaton, "Stool form scale as a useful guide to intestinal transit time," Scandinavian Journal of Gastroenterology, vol. 32, no. 9, pp. 920-924, 1997. 